\section{Quasi-phase-matched generation of coherent extreme-ultraviolet light}

A. Paul ${ }^{\star}$, R. A. Bartels ${ }^{\star}$, R. Tobey ${ }^{\star}$, H. Green ${ }^{\star}$, S. Weiman ${ }^{\star}$, I. P. Christov $\dagger$, M. M. Murnane ${ }^{\star}$, H. C. Kapteyn ${ }^{\star} \&$ S. Backus ${ }^{\star}$

* Department of Physics and JILA, University of Colorado and National Institute of Standards and Technology, Boulder, Colorado 80309-0440, USA

$\dagger$ Department of Physics, Sofia University, Sofia 1164, Bulgaria

High-harmonic generation is a well-known method of producing coherent extreme-ultraviolet (EUV) light, with photon energies up to about $0.5 \mathrm{keV}$ (refs 1,2 ). This is achieved by focusing a femtosecond laser into a gas, and high harmonics of the fundamental laser frequency are radiated in the forward direction ${ }^{3,4}$. However, although this process can generate high-energy photons, efficient high-harmonic generation has been demonstrated only for photon energies of the order $50-100 \mathrm{eV}$ (ref. 5). Ionization of the gas prevents the laser and the EUV light from propagating at the same speed, which severely limits the conversion efficiency. Here we report a technique to overcome this problem, and demonstrate quasi-phase-matched frequency conversion of laser light into EUV. Using a modulated hollow-core waveguide to periodically vary the intensity of the laser light driving the conversion, we efficiently generate EUV light even in the presence of substantial ionization. The use of a modulated fibre shifts the energy spectrum of the high-harmonic light to significantly higher photon energies than would otherwise be possible. We expect that this technique could form the basis of coherent EUV sources for advanced lithography and highresolution imaging applications. In future work, it might also be possible to generate isolated attosecond pulses.

Advances in nonlinear optics have greatly expanded the utility of the laser ${ }^{6}$. Using nonlinear crystals and frequency-conversion techniques such as frequency doubling ${ }^{7}$ and quasi-phase matching $(\mathrm{QPM})^{8-10}$, laser light at one wavelength can be converted to another. In nonlinear optics, efficient conversion requires that the process be phase matched ${ }^{6}$. As a pump beam propagates through a medium, the nonlinear response leads to generation of a signal beam at a different wavelength. If the two waves travel with the same phase velocity, the nonlinear response continues to add constructively to the signal beam, leading to a bright, 'phase-matched' output signal at a new wavelength. If a process cannot be phase matched, the phase between the signal field and the nonlinear response will eventually reach $180^{\circ}$, resulting in destructive interference and back-conversion into the fundamental field. The propagation distance at which destructive interference occurs is defined as the coherence length.

Phase matching in the visible is often accomplished using anisotropic crystals. Here, the signal and pump beams propagate with different polarizations, taking advantage of the birefringence of the medium to equalize propagation velocities. However, other approaches are possible. In 1962, shortly after the birth of nonlinear optics, Armstrong et al. ${ }^{11}$ proposed "phase corrective schemes", later termed QPM schemes. In QPM, a phase mismatch is periodically 'corrected', with a periodicity corresponding to twice the coherence length. For example, by reversing the crystal orientation of the medium, the polarity of the nonlinear response is reversed just before destructive interference occurs. However, practical implementation of this concept awaited the development of crystal-poling techniques in the mid-1990 $\mathrm{s}^{8-10}$.

Unfortunately, reliance on solid materials for nonlinear frequency conversion has severely limited nonlinear optical techniques for generating very-short-wavelength light. Most solid materials are transparent only at wavelengths greater than $150-200 \mathrm{~nm}$. Thus, the generation of coherent light at EUV wavelengths must take place in a gas $^{3,4}$, where traditional phase-matching techniques do not work. Nevertheless, phase-matched frequency conversion to the EUV is still possible, and leads to the generation of fully spatially ${ }^{12}$ and temporally ${ }^{13}$ coherent beams. In past work, we demonstrated the generation of phase-matched high-harmonic generation (HHG) over an extended propagation range, by confining the light in a gasfilled hollow waveguide ${ }^{5,14}$. The effect of the neutral gas index of refraction on the pump light (decreasing the phase velocity of the pump) can be counterbalanced by waveguide dispersion and ionization (increasing the phase velocity of the pump), making it possible to equalize the propagation velocity of the pump and signal. However, this approach to phase matching is limited to relatively low EUV photon energies. Higher EUV photon energies are generated at higher levels of ionization, where the phase velocity of the pump beam is too fast to be phase matched using any technique demonstrated to date. Thus, although HHG can generate photons with energy of about $500 \mathrm{eV}$ (refs 1,2), efficient phasematched HHG has been demonstrated only at photon energies of $\leq 50-90 \mathrm{eV}$. This prevents the use of this source for applications such as in vitro imaging of small cellular structures, which requires EUV light in the 'water window' $(\sim 300 \mathrm{eV})$ region of the spectrum. Coherent sources at the $13-\mathrm{nm}$ wavelength of interest to EUV lithography would also benefit from increased efficiency.
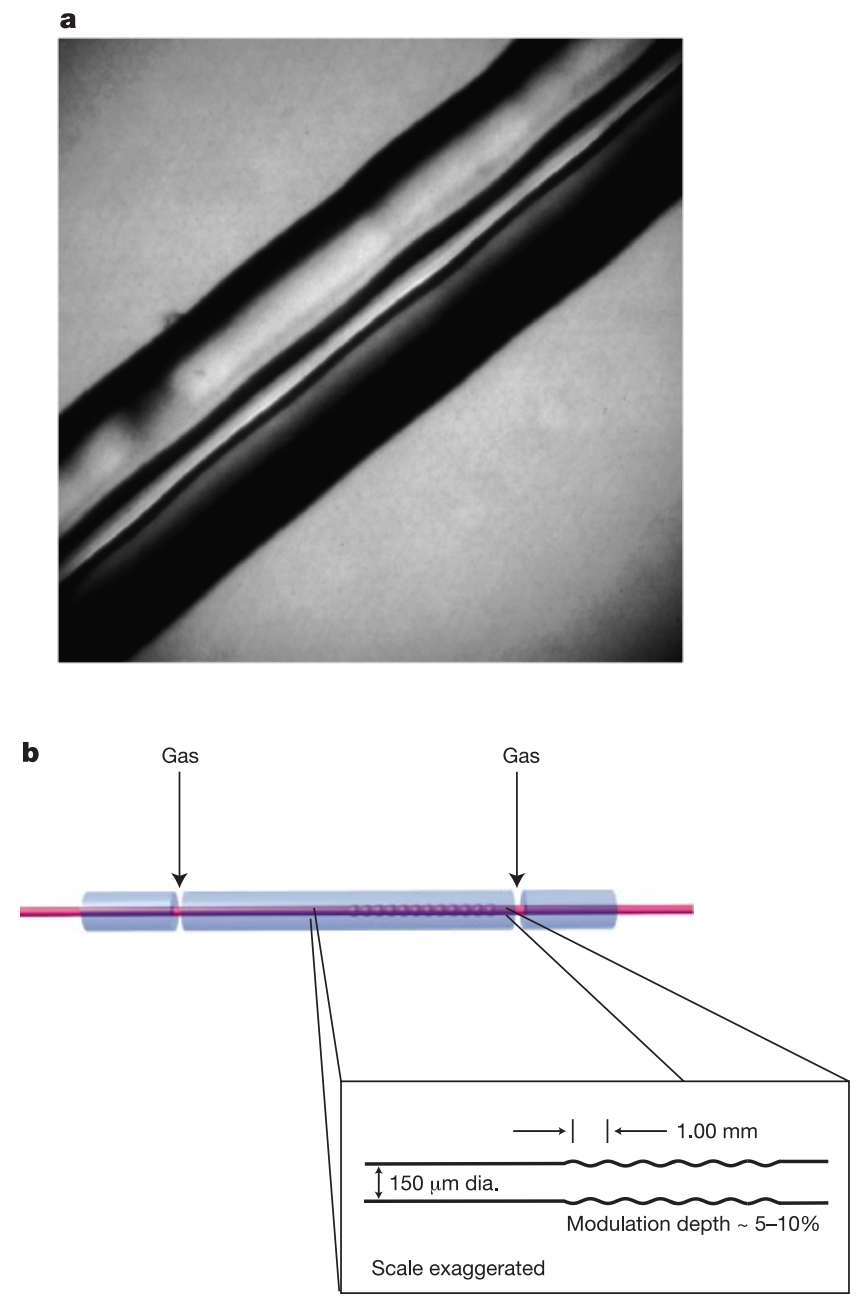

Figure 1 Modulated hollow-core fibres used to implement quasi-phase matching in the EUV. a, Picture of hollow-core modulated fibre, $\mathbf{b}$, Details of modulated fibre with periodicity of $1 \mathrm{~mm}$, inner diameter of $150 \mu \mathrm{m}$, and modulation depth of $10 \mu \mathrm{m}$. 
Here we demonstrate that this limitation can be overcome by applying QPM techniques to HHG. Using a hollow-core waveguide with a periodically modulated diameter, the energy range over which we efficiently generate high harmonics can be increased significantly. The periodic structure modulates the generation of high harmonics and restricts HHG to regions with favourable constructive interference. It may also allow for the generation of isolated attosecond pulses. This work shows that sophisticated concepts of nonlinear optical 'photonics' and engineered structures can be applied even to the extreme nonlinear optics of HHG. It also complements our past work using optimally shaped light pulses to selectively optimize $\mathrm{HHG}^{13}$. Both works show that the highly nonlinear nature of the HHG process, rather than being a limitation, in fact allows for new control mechanisms that are simply not possible in conventional nonlinear optics.

In our experiment, 25-fs-duration pulses from a high-repetitionrate $(2-5 \mathrm{kHz}, 1 \mathrm{~mJ}$ per pulse) Ti:sapphire laser system operating at $760 \mathrm{~nm}$ (ref. 15) were focused into $150-\mu \mathrm{m}$-diameter hollow fibres
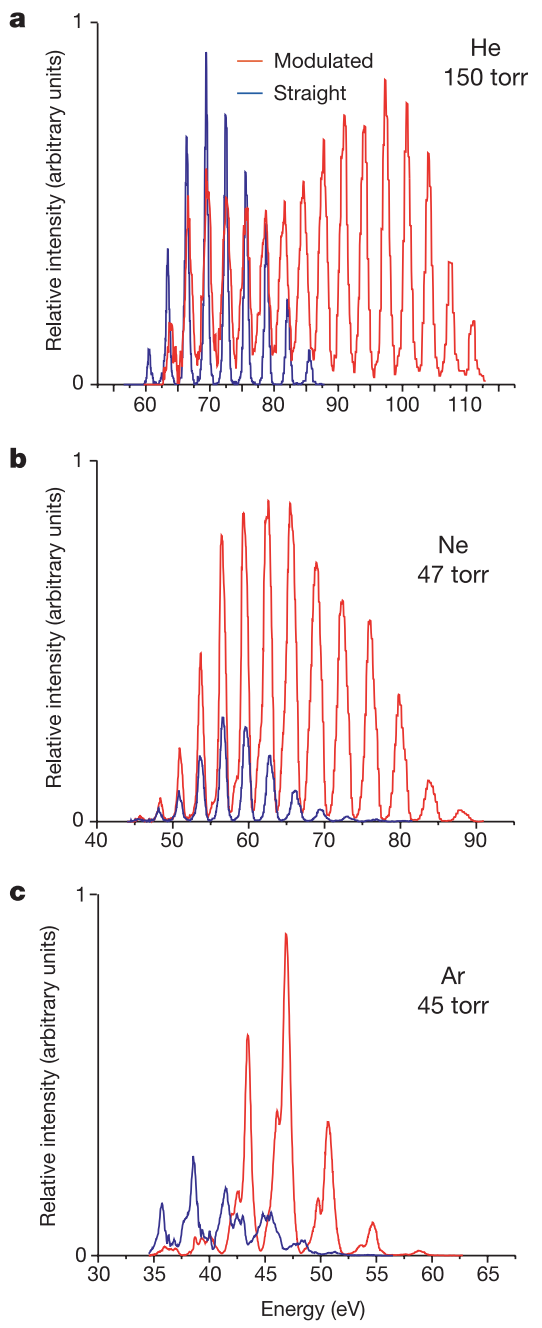

Figure 2 Experimentally measured HHG spectra for straight (blue) and modulated (red) fibres. Data are shown for He gas (a), Ne gas (b) and $\operatorname{Ar}$ gas (c), at pressures of 150 torr, 47 torr and 45 torr, respectively. In this case, the modulated section was $1 \mathrm{~cm}$ in length, with $1 \mathrm{~mm}$ periodicity, placed near the end of the fibre. The measured flux (non-optimized) corresponds approximately to $1 \mathrm{~nJ}$ per harmonic per pulse for Ar, and $20 \mathrm{pJ}$ per harmonic per pulse for $\mathrm{He}$ at repetition rates of $2 \mathrm{kHz}$. These numbers are expected to increase when sufficient laser intensity is available to push the $\mathrm{HHG}$ spectra into the transparency region of the gas, and when the lengths of the modulated fibres are optimized. filled with various gases. Some of the waveguides were periodically modulated in diameter to modulate the laser intensity inside the fibre. The modulated fibres used in this experiment were produced using precision glass-blowing techniques starting with a straight hollow-core fibre (also $150 \mu \mathrm{m}$ in diameter). The modulations were approximately sinusoidal, with a period of $1-0.5 \mathrm{~mm}$, and a radial depth of the order of $10 \mu \mathrm{m}$, corresponding to a $13 \%$ modulation of the fibre radius (Fig. 1).

Figure 2 shows the phase-matched HHG spectrum from $\mathrm{He}, \mathrm{Ne}$ and Ar gas for straight (blue) and modulated (red) fibres. For all cases, the flux measured from the QPM fibres is significantly greater (by factors of 2-5) than that measured from straight fibres. Most significantly, the modulated fibre increases the brightness of higher harmonic orders by at least two orders of magnitude. In the case of $\mathrm{He}$, for the straight fibre the HHG comb spans an energy range from 60 to $80 \mathrm{eV}$, with a spectral peak at $68 \mathrm{eV}$ (Fig. 2a, blue trace). For the modulated fibre, the spectral peak shifts by $27 \mathrm{eV}$, from 68 to $95 \mathrm{eV}$ (Fig. 2a, red), while the QPM harmonic comb also spans a broader energy range from 63 to $112 \mathrm{eV}$. Note that the wavelength region over which we achieve QPM easily includes the 94-eV $(13 \mathrm{~nm}$ wavelength) region of interest for the next generation of EUV lithographies. In the case of $\mathrm{Ne}$, use of a modulated fibre extends the high-flux harmonic emission from 70 to $90 \mathrm{eV}$, while significantly increasing the flux (Fig. 2b). In the case of Ar, the HHG spectrum from a straight fibre consists of a comb of phase-matched harmonics, peaked around $37 \mathrm{eV}$ (Fig. 2c, blue). For the modulated fibre (Fig. 2c, red), the HHG output spectral peak shifts to $47 \mathrm{eV}$, while the flux increases significantly. We also investigated the HHG emission from Ar when the laser intensity was reduced by a factor of three. In this case (Fig. 3), the characteristic 'harmonics' merge into an almost smooth continuum of both even and odd orders. Moreover, the spectral bandwidth of the merged peaks for the QPM case corresponds to a single 250-as-duration EUV pulse, assuming a time-bandwidth limit (Fig. 3 inset).

We also investigated HHG from longer $(2.5 \mathrm{~cm})$ modulated fibres with different modulation periods. Figure 4 shows the experimen-

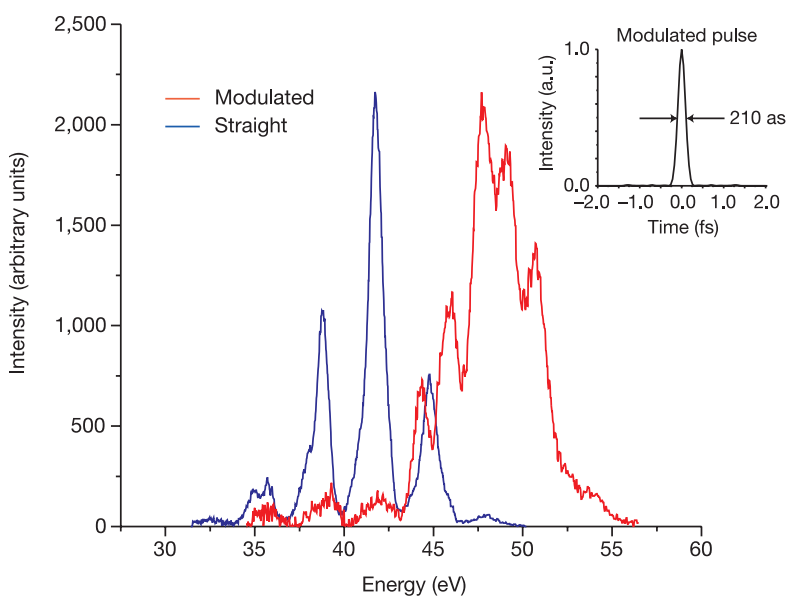

Figure 3 Experimentally measured HHG spectra from Ar for straight (blue) and modulated (red) fibres, at lower intensities than Fig. 2c, and at a pressure of 25 torr. In this case, the modulated section was $1 \mathrm{~cm}$ in length, with $1 \mathrm{~mm}$ periodicity, placed in the centre of the fibre. Inset, Fourier transform of the HHG emission from the modulated fibre, predicting the generation of a 250-as pulse, provided that the phase is flat. The reason for the different characteristic QPM spectra from Ar in Fig. 2c and Fig. 3 results from the use of different laser intensities and gas pressures in the two cases, resulting in a different temporal phase-matching window. Thus, the effect of QPM is evident in both cases, and has the potential for generating isolated attosecond pulses under the correct conditions. a.u., arbitrary units. 
tally measured HHG spectra from He for three different periodicities of the modulated fibres. As the modulation period is reduced from $1 \mathrm{~mm}$, to $0.75 \mathrm{~mm}$, to $0.5 \mathrm{~mm}$, the high-energy cut-off increases from 112 to $175 \mathrm{eV}$. This cut-off is limited by the available laser intensity, and is expected to increase significantly when longer modulated sections, lower pressures, shorter laser pulses, or higher laser intensities are used.

To understand our results, we use previous theoretical predictions of the potential utility of QPM frequency conversion in the EUV $^{16,17}$. HHG is extremely sensitive to intensity; thus a periodic modulation in the intensity of the driving laser will modulate the HHG emission. Generation of the highest harmonic orders is 'turned off' when the fibre bulge increases the mode diameter in the fibre, thus preventing back-conversion of the EUV light. Therefore HHG will be quasi-phase matched when the period of the modulation matches the period of the phase mismatch between the pump and signal (that is, twice the coherence length). We also note that two other experimental works used QPM in low-order harmonic generation in gases ${ }^{18,19}$. However, their approach is not applicable to the EUV. Other proposed schemes, such as suppressing HHG with interfering beams, may be applicable in the EUV ${ }^{20}$.

An intuitive picture of our experimental results can be obtained in the perturbative approach. The signal corresponding to the $q$ th harmonic $\left(E_{q_{\omega}}(L)\right)$ after propagation through a generation medium of length $\mathrm{L}$ is given by:

$$
E_{q \omega}(L) \approx \int_{o}^{L} E_{\omega}^{q}(z) d(z) \mathrm{e}^{i \Delta k z} \mathrm{~d} z
$$

where $E_{\omega}(z)$ is the laser field, $d(z)$ is the effective nonlinear coefficient for HHG, and $\Delta k=q k_{\omega}-k_{q_{\omega}}$ is the net phase mismatch between the fundamental and harmonic field. In the absence of phase matching or quasi-phase matching, the rapid $e^{i \Delta k z}$ phase term will cause the integral in equation (1) to average to zero. This integral will be non-zero if either $\Delta k=0$, corresponding to traditional phase matching in the visible or EUV, or if $d(z) \approx$ $\cos \left(\Delta k_{\mathrm{M}} z\right)$, corresponding to traditional QPM in the visible. For

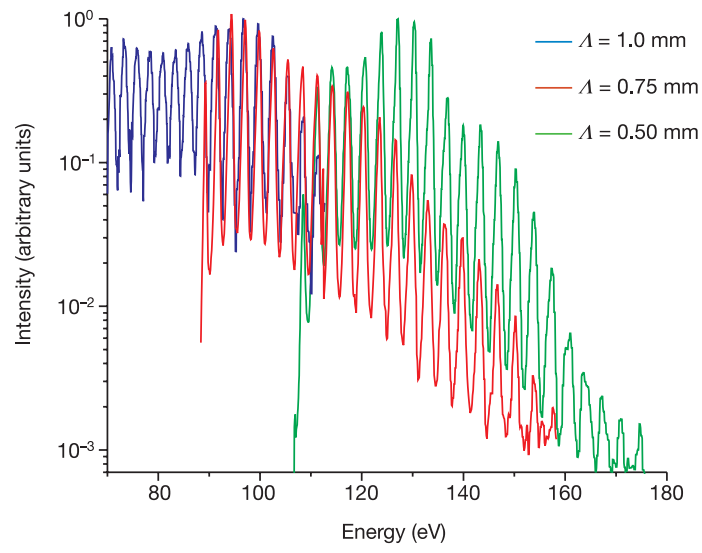

Figure 4 Experimentally measured HHG spectra (log scale) from He for three different periodicities of the modulated fibres, each $2.5 \mathrm{~cm}$ in length. Blue, $1 \mathrm{~mm}$ periodicity $(\Lambda)$; red, $0.75 \mathrm{~mm}$ periodicity; green, $0.5 \mathrm{~mm}$ periodicity. All spectra were taken through two $0.2-\mu \mathrm{m}$ zirconium filters, which rejected the laser light. The gas pressure was 111 torr, and the laser intensity was about $5 \times 10^{14} \mathrm{~W} \mathrm{~cm}^{-2}$. The different curves were taken at different positions of the EUV grating in the EUV spectrometer, owing to the finite spectral region that can be captured simultaneously on the CCD. Therefore, the cut-off at low energy is artificial, limited by the spectral window. The high-energy cut-off is limited by the available laser intensity. It is expected to increase significantly when longer modulated sections, lower pressures, shorter laser pulses (10 fs), or higher laser intensities are used. the latter, the sign on the nonlinear coefficient $d(z)$ is modulated periodically using periodically poled materials ${ }^{8-10,21}$. The modulation period $\Lambda$ (where $\Delta k_{\mathrm{M}}=2 \pi / \Lambda$ ) is chosen to be equal to the coherence length of the signal, that is, the distance over which the fundamental and harmonic fields undergo a phase slip of $\pi$, to ensure that the harmonic signal from different regions in the interaction length always adds in phase.

In our work, a new type of QPM can be implemented in the EUV where the signal wave itself is modulated periodically, that is, $E_{\omega}^{q}(z) \approx \cos \left(\Delta k_{\mathrm{M}} z\right)$ and $d$ is independent of $z$. The periodic QPM structure can modulate the generation of high harmonic orders in several ways. Harmonics near cut-off that require higher laser intensity for generation can be turned on and off by the modulation. However, other effects (such as the periodic evolution of the phase of the driving pulse) can also contribute to a periodic modulation of the harmonic generation process. In fact, virtually any periodic change in the character of the harmonic emission can allow QPM to operate, particularly in a very-high-order nonlinear process where very small phase changes can dramatically change the output. ${ }^{13}$

The significance of the quasi-phase matching discussed here is that it permits phase matching of HHG at higher ionization levels and hence higher photon energies than previously possible. This can be made apparent by calculating the coherence length that results from ionization of the gas. The plasma-induced change in index of refraction corresponds to a phase mismatch in equation (1) of $\Delta k_{\text {plasma }} \approx q n_{\mathrm{e}} e^{2} \lambda / 4 \pi m_{\mathrm{e}} \epsilon_{0} c^{2}$, where $\lambda$ is the laser wavelength, $e$ is the charge of the electron, $m_{\mathrm{e}}$ is the mass of the electron, $\epsilon_{0}$ is the vacuum permittivity, and $n_{\mathrm{e}}$ is the electron density. For fully ionized argon at a pressure of 1 torr, and for $q=29, n_{\mathrm{e}}=3.3 \times 10^{16} \mathrm{~cm}^{-3}$, giving $\Delta k \approx 23 \mathrm{~cm}^{-1}$. Therefore, the coherence length, $L_{\mathrm{c}}$, given by $L_{\mathrm{c}}=\pi / \Delta k$, is $1.4 \mathrm{~mm}$. Thus, very substantial levels of ionization can be compensated for using the QPM technique demonstrated here, with experimentally realizable modulation periods in the millimetre range. In our experiments, because we can modulate at most a 2.5-cm-long section of fibre, we used higher pressures (30 torr). This implies that the $1 \mathrm{~mm}$ modulation period compensates approximately for an additional $4 \%$ fractional ionization at harmonic order 29. This is to be compared with a straight fibre where phase matching occurs at ionization levels of $2-4 \%$; thus, QPM more than doubles the allowable level of ionization in this case. For $\mathrm{He}$, the effect of the QPM is greater, because the fractional ionization at which high harmonics are efficiently generated is much lower $(<0.6 \%$ for $q=61$, for example). Therefore, a small change in allowable ionization results in a large change in the energy of the highest phase-matched harmonic order. Finally, by decreasing the modulation period from 1 to $0.5 \mathrm{~mm}$ (Fig. 4), the amount of ionization that can be compensated increases, allowing for even higher harmonic orders to be phase matched. Furthermore, by increasing the length of the modulated fibre and reducing the pressure, very high levels of ionization might be compensated for, leading to the generation of very-high-order harmonics, perhaps even from fully ionized gasses. This approach also leads to a significant increase in output flux, because the transparency of the gas is increasing with increasing photon energy over the range we investigate, while the effective nonlinearity is not changing rapidly. For $\mathrm{He}$ and $\mathrm{Ne}$ for example, the absorption length is increasing with increasing harmonic photon energies. Therefore, we observe both higher harmonic orders and increased overall flux in the data of Fig. 2.

The QPM structure also suggests the generation of isolated attosecond pulses, even though the driving pulse is nearly two orders of magnitude longer than the generated pulse. This behaviour results from the fact that a dynamic, plasma-induced phase dominates the phase-matching conditions. As a laser beam propagates through a region of space, the level of ionization increases monotonically with time. At a critical ionization level, the phasematching conditions will be optimal, and the bulk of the observed 
signal originates from a short window in time within the driving pulse. In QPM, this critical ionization level is higher, and occurs closer in time to the peak of the pulse if the optimal laser intensity is used. Here, the ionization rate is highest, resulting in a very fast 'sweep' of the phase-matching conditions through the optimum. Simple calculations show that this time window can be significantly shorter than for the equivalent straight fibre, and may limit the HHG emission to a single half-cycle of the driving laser. This phenomenon of time-gating of HHG by means of rapidly varying phase-matching conditions is new, and is distinct from the concept of time-gating using intensity variations in a short, sub-10-fs pulse $\mathrm{e}^{22,23}$.

Finally, by using either higher intensity laser pulses, longer modulated sections, lower pressures, or shorter (10 fs) pulses, our calculations show that it should be possible to extend this work to phase match HHG at wavelengths well below $4.4 \mathrm{~nm}$. This wavelength is in the 'water window' region of the spectrum that is relevant to high-contrast biological imaging; HHG has been shown to reach these short wavelengths, but only under inefficient non-phase-matched conditions ${ }^{1,2}$. We expect that this phasematched EUV source will thus make possible element-specific spectroscopies and microscopies with ultrahigh spatial and temporal resolution.

Received 23 July; accepted 15 October 2002; doi:10.1038/nature01222.

1. Chang, Z. H., Rundquist, A., Wang, H. W., Murnane, M. M. \& Kapteyn, H. C. Generation of coheren soft X rays at $2.7 \mathrm{~nm}$ using high harmonics. Phys. Rev. Lett. 79, 2967-2970 (1997).

2. Spielmann, C. et al. Generation of coherent X-rays in the water window using 5-femtosecond laser pulses. Science 278, 661-664 (1997).

. McPherson, A. et al. Studies of multiphoton production of vacuum-ultraviolet radiation in the rare gasses. J. Opt. Soc. Am. B 4, 595-601 (1987).

4. Ferray, M. et al. Multiple-harmonic conversion of $1064 \mathrm{~nm}$ radiation in rare gasses. J. Phys. B 21, L31-L35 (1987).

5. Rundquist, A. et al. Phase-matched generation of coherent soft X-rays. Science 280, 1412-1415 (1998).

6. Bloembergen, N. Nonlinear Optics (World Scientific, River Edge, NJ, 1996).

7. Franken, P. A., Hill, A. E., Peters, C. W. \& Weinreich, G. Generation of optical harmonics. Phys. Rev. Lett. 7, 118-119 (1961)

8. Miller, G. D. et al. $42 \%$-efficient single-pass $\mathrm{cw}$ second-harmonic generation in periodically poled lithium niobate. Opt. Lett. 22, 1834-1836 (1997).

9. Fejer, M. M., Magel, G. A., Jundt, D. H. \& Byer, R. L. Quasi-phase-matched 2nd harmonic-generation - Tuning and tolerances. IEEE J. Quant. Electron. 28, 2631-2654 (1992).

10. Zhu, S., Zhu, Y. Y. \& Ming, N. B. Quasi-phase-matched third-harmonic generation in a quasi-periodic optical superlattice. Science 278, 843-846 (1997).

11. Armstrong, J. A., Bloembergen, N., Ducuing, J. \& Pershan, P. S. Interactions between light waves in a nonlinear dielectric. Phys. Rev. A 127, 1918-1939 (1962).

12. Bartels, R. A. et al. Fully spatially coherent EUV beams generated using a small-scale laser. Science 297, 376-378 (2002).

13. Bartels, R. et al. Shaped-pulse optimization of coherent soft X-rays. Nature 406, 164-166 (2000).

14. Durfee, C. G. et al. Phase matching of high-order harmonics in hollow waveguides. Phys. Rev. Lett. 83, 2187-2190 (1999)

15. Backus, S. et al. High-efficiency, single-stage 7-kHz high-average-power ultrafast laser system. Opt. Lett. 26, 465-467 (2001)

16. Christov, I. P., Kapteyn, H. C. \& Murnane, M. M. Quasi-phase matching of high-harmonics and attosecond pulses in modulated waveguides. Opt. Express 7, 362-367 (2000).

17. Shkolnikov, P. L., Lago, A. \& Kaplan, A. E. Optimal quasi-phase-matching for high-order harmonic generation in gases and plasmas. Phys. Rev. A 50, R4461-R4464 (1994).

18. Lange, H. R. et al. High-order harmonic generation and quasiphase matching in xenon using selfguided femtosecond pulses. Phys. Rev. Lett. 81, 1611-1613 (1998).

19. Misoguti, L. et al. Generation of broadband VUV light using third-order cascaded processes. Phys. Rev. Lett. 87, 013601-013604 (2001).

20. Voronov, S. L. et al. Control of laser high-harmonic generation with counterpropagating light. Phys. Rev. Lett. 87, 133902-133905 (2001).

21. Somekh, S. \& Yariv, A. Phase matching by periodic modulation of the nonlinear optical properties. Opt. Commun. 6, 301-304 (1972).

22. Christov, I. P., Murnane, M. M. \& Kapteyn, H. C. High-harmonic generation of attosecond pulses in the "single-cycle" regime. Phys. Rev. Lett. 78, 1251-1254 (1997).

23. Hentschel, M. et al. Attosecond metrology. Nature 414, 509-513 (2001).

Acknowledgements This work was supported by the National Science Foundation and the Department of Energy.

Competing interests statement The authors declare competing financial interests: details accompany the paper on Nature's website (http://www.nature.com/nature)

Correspondence and requests for materials should be addressed to M.M.M. (e-mail: murnane@jila.colorado.edu).

\section{Electroluminescent device with reversible switching between red and green emission}

\author{
S. Welter ${ }^{\star}$, K. Brunner $\dagger$, J. W. Hofstraat ${ }^{\star} \dagger$ \& L. De Cola ${ }^{*}$
}

${ }^{*}$ Molecular Photonics Group, University of Amsterdam, Nieuwe Achtergracht 166, 1018 WV Amsterdam, The Netherlands

$\dagger$ Philips Natuurkundig Laboratorium, Prof. Holstlaan 4, 5656 AA Eindhoven, The Netherlands

Research on new materials for organic electroluminescence has recently focused strongly on phosphorescent emitters ${ }^{1-3}$, with the aim of increasing the emission efficiency and stability. Here we report the fabrication of a simple electroluminescent device, based on a semiconducting polymer combined with a phosphorescent complex, that shows fully reversible voltagedependent switching between green and red light emission. The active material is made of a polyphenylenevinylene (PPV) derivative molecularly doped with a homogeneously dispersed dinuclear ruthenium complex, which fulfils the dual roles of triplet emitter and electron transfer mediator. At forward bias $(+4 \mathrm{~V})$, the excited state of the ruthenium compound is populated, and the characteristic red emission of the complex is observed. On reversing the bias $(-4 \mathrm{~V})$, the lowest excited singlet state of the polymer host is populated, with subsequent emission of green light. The mechanism for the formation of the excited state of the PPV derivative involves the ruthenium dinuclear complex in a stepwise electron transfer process that finally leads to efficient charge recombination reaction on the polymer.

The work of Bard and co-workers ${ }^{4}$ on the electroluminescence of ruthenium complexes in solution prepared the ground for the design and development of solid-state devices based on metal complexes. The Ru complexes form an interesting class of electroluminescent materials. Red emission, centred around $620 \mathrm{~nm}$, high external efficiency ${ }^{5-7}$ at low voltages, easy synthesis and good processability are attractive properties of these complexes ${ }^{8}$, which make them eminent candidates for application as dopant in emissive polymer layers. The polypyridine $\mathrm{Ru}$ complexes are intrinsically charged. The mechanism associated with their electroluminescence is based on charge injection across the electrodes via an electrochemical redox pathway, and therefore is independent of the work function of the electrodes. Such devices are referred to as light-emitting electrochemical cells ${ }^{9}$.

A dinuclear ruthenium complex, referred to here as $\left[\mathrm{Ru}(\mathrm{ph})_{4^{-}}\right.$ $\mathrm{Ru}]^{4+}$ (see below for full structure), containing two $\mathrm{Ru}(\mathrm{bpy})_{3}^{2+}$ moieties (where bpy is $2,2^{\prime}$-bipyridine) and four phenylene (ph) units as spacers between the two ruthenium centres, was mixed $(\mathrm{Ru} /$ PPV mass ratio 1:5) with a green-emitting polyphenylenevinylene derivative, poly(2-( $m$-3,7-dimethyloctyloxy-phenyl)-p-phenylenevinylene, referred to here as 'PPV'. The light-emitting polymer and the dinuclear ruthenium complex are applied such that they form a homogeneous, amorphous layer on a scale that can be observed by visual inspection and optical luminescence microscopy of the layer. For reference purposes and to assess the proposed mechanism (see below), three other complexes, referred to here as $\left[\mathrm{Ru}(\mathrm{bpy})_{3}\right]^{2+},\left[\mathrm{Ru}(\mathrm{ph})_{4}\right]^{2+}$ and $\left[\mathrm{Ru}-\mathrm{Ada}_{2}-\mathrm{Ru}\right]^{4+}$ (ref. 10), were used (see below for full structures). No lateral phase separation is observed in the film formed by the polymer doped with the metal complexes (as measured, for example, by secondary ion mass spectrometry).

The highest occupied molecular orbital (HOMO) levels of the polymer and of the ruthenium compound are almost isoenergetic, whereas the lowest unoccupied molecular orbital (LUMO) of the Ru complex is typically about $0.5 \mathrm{eV}$ lower in energy than that of the 